\title{
A procedure for adaptive evaluation of numerical and experimental data
}

\author{
J. Krok ${ }^{1}$, M. Stanuszek ${ }^{2} \&$ J. Wojtas ${ }^{2}$ \\ ${ }^{1}$ Institute of Computer Methods in Mechanics, \\ Cracow University of Technology, Cracow, Poland \\ ${ }^{2}$ Institute of Computer Modeling, \\ Cracow University of Technology, Cracow, Poland
}

\begin{abstract}
The work addresses extended formulation of a new approach proposed to control error of experimental data. It includes: development of postprocessing techniques to approximate data given in a discrete form, a'posteriori error estimation (evaluation) of measured data and definition of reliability index of experimental data. Theoretical consideration and numerical analysis are based on the Adaptive Meshless Finite Difference (MFDM) approach.
\end{abstract}

Keywords: meshless FDM, experimental data approach and smoothing, a'posteriori error estimation of experimental data, adaptive methods.

\section{Introduction}

Almost all numerical procedures of computational mechanics consist of the discretization process in which the continuous model is transformed into a discrete one. The discretization process is made as well within experimental setup and constitutes the key point of the computer simulation. It has a strong influence on the exactness, efficiency, generality and usefulness of obtained results. Correct discretization strategy and control of the discretization process very often decide whether the solution of analysed task is obtained or not.

One may investigate the exactness of calculations (simulation) i.e.: how to measure the error due to numerical simulation (coming from the discretization process), minimize obtained error and effectively eliminate it. The question of verification applies here as well, i.e. if one can assess received results by the 
theoretical consideration (using available classical solution), will be the proposed procedure of estimation safe and applicable to other difficult and complex task as for example estimation of the different errors of the experimental data.

In present work the efficiency of a new, coherent concept of a'posteriori error estimation of experimental or numerical (results of FEM (Finite Element Method) or FDM (Finite Difference Method)) data, together with estimation of the mesh density, taking into consideration the equal error distribution, is considered. Approach using the discrete function and MWLS (Moving Weighted Least Square) method with constrains, defined by the differential theory equations (e.g. equations of equilibrium, boundary conditions etc.) was applied. Several ways of error estimation as well as experimental points distribution were proposed. The suggested procedures of error estimation and density prediction of experimental points distribution were tested on solution of certain mechanical problems.

The introduced approach relates to the so-called, problem - oriented a'posteriori constructed estimators. The special attention was given to define an error functional with additional conditions i.e. constrains. Such an approach makes the estimation of the error of separate components possible (e.g. one component of strain or stress state or one element of body potential energy [3] may be analyzed). Mainly, error estimators base on the behaviour of the total energy, recording and responding to its change (e.g. FEM estimators of Zienkiewicz-Zhu [9]). Followed by the change of the total energy of the deformed body a part of the information on change of its components is lost.

The problem of approach and error estimation of experimental and/or numerical data was commonly considered by many researchers during the last three decades. Among these the paper of Karmowski and Orkisz [1] provide first concept of coupling of analytical as well as physical information on the case within the solution of the problem. Following this idea, a concept of physically based local - global approach was introduced. Simultaneously Lukasiewicz and Stanuszek [5, 6] developed their own concept of filtering and verification of numerical as well as experimental data. They formulated an error functional, as a combination of least square approximation of error with constrains in the form of theoretical equation approach. Presented idea turned out very attractive, especially in problems of mechanics, where equations of equilibrium and continuity as well as boundary conditions have to be satisfied.

The extension of the idea of physically based approach to experimental data was delivered recently by Magiera [7], where certain non-statistical considerations for a'posteriori estimation were presented.

Next important stage in constructing a correct estimator of experimental data was achieved by Krok and Wojtas. They have defined [2, 4] the density distribution of experimental points as the function of error distribution. Several ideas on converting the data errors to the node density distribution were also considered there. Current paper follows and extends the approaches presented in those two works. 


\section{Formulation of the problem}

\subsection{General remarks}

During the process of collecting experimental and/or calculating numerical data several typical situations may be encountered:

a) it is impossible to avoid the errors of measurement or calculation, in other words it is impossible to obtain the results of such processes without errors,

b) zones with large gradients of measured and/or calculated function, indicate the domains in which high density of points is required,

c) application of data smoothing (approach technology) lowers the amount of information available to assess the results of FEM and/or FDM analysis.

The user is strongly encouraged to make use of all available physical, theoretical as well as numerical information to estimate the error distribution and based on this, predict the corrected node distribution.

\subsection{Definition of error functional}

In order to begin, let us assume a "raw" data vector $\widetilde{\mathbf{u}}\left\{\widetilde{\mathbf{u}}_{1}, \ldots, \widetilde{\mathrm{u}}_{n}\right\}$ with components being the values measured and/or numerically calculated at discrete $n$ points of a grid $\theta$. Additional information on the model of the analyzed system is provided by constraints and can be presented as the following set of equations:

$$
\mathbf{H u}=\mathbf{f}
$$

in which $\mathbf{H}_{[n \times k]}$ is the matrix resulting from the application of constraints and $\mathbf{u}$ is a vector of unknown, corrected data $\mathbf{u}\left\{\mathrm{u}_{1}, \ldots . \mathrm{\mu}_{m}\right\}$, usually located at $m$ points of a grid $\gamma$ differing form grid $\theta$; vector $\mathbf{f}\left\{\mathrm{f}_{1}, \ldots . \mathrm{f}_{k}\right\}$ represents the right side of the constraints equations.

Figure 1: The considered domain and points: $\mathrm{o}$ - with measured data $\widetilde{\mathbf{u}}-$ set $\theta, \Delta$ - with unknown data $\mathbf{u}$ - set $\gamma$. 
When the grid of points with measured or calculated data differs from the grid of verified (unknown) data $(\theta \neq \gamma)$, one has to approximate the vector $\mathbf{u}\left\{\mathrm{u}_{1}, \ldots . \mathrm{u}_{m}\right\}$ using for example the MWLS (Moving Weighted Least Square) technique:

$$
\overline{\mathbf{u}}=\mathbf{A} \mathbf{u}
$$

where $\overline{\mathbf{u}}\left\{\overline{\mathrm{u}}_{1}, \ldots ., \overline{\mathrm{u}}_{n}\right\}$ are the unknown values of verified data on the grid $\theta$ used for measured and/or calculated data. A matrix $\mathbf{A}_{[\mathrm{m} \times k]}$ results from the procedure (MWLS) for verified $(\gamma)$ and measured/calculated $(\theta)$ points which in general are different.

The constraints described by eq. (1) can be satisfied in a sense of the least squares technique. Therefore the calculated value of $\mathbf{u}$ has to minimize the following error function $R$

$$
R(\mathbf{u}, \boldsymbol{\lambda})=\frac{1}{2}(\mathbf{A} \mathbf{u}-\widetilde{\mathbf{u}})^{2}+(\mathbf{H} \mathbf{u}-\mathbf{f})^{T} \boldsymbol{\lambda}
$$

where $\lambda$ represents a vector of Lagrange multipliers. In other words, the vector $\mathbf{u}$ should satisfy the equality constraints (1) and be as close as possible to the numerically calculated values $\widetilde{\mathbf{u}}$. The set of equations (3) has to be represented by a discrete model of the system in terms of finite difference operators constructed on irregular grids based on the approach (2). Based on the standard minimizing procedure with respect to $\mathbf{u}$ and $\lambda$ vectors

$$
\frac{\partial R}{\partial \mathbf{u}}=\mathbf{A}^{T} \mathbf{A} \mathbf{u}-\mathbf{A}^{T} \widetilde{\mathbf{u}}+\mathbf{H}^{T} \boldsymbol{\lambda}=0, \quad \frac{\partial R}{\partial \boldsymbol{\lambda}}=\mathbf{H u}-\mathbf{f}=0
$$

one can get two separated sets of linear equations leading to $\mathbf{u}$ and $\lambda$

$$
\begin{gathered}
\boldsymbol{\lambda}=\left(\mathbf{H}\left(\mathbf{A}^{T} \mathbf{A}\right)^{-1} \mathbf{H}^{T}\right)^{-1} \mathbf{H}\left(\mathbf{A}^{T} \mathbf{A}\right)^{-1} \mathbf{A}^{T} \widetilde{\mathbf{u}}-\left(\mathbf{H}\left(\mathbf{A}^{T} \mathbf{A}\right)^{-1} \mathbf{H}^{T}\right)^{-1} \mathbf{f} \\
\mathbf{u}=\left(\mathbf{A}^{T} \mathbf{A}\right)^{-1} \mathbf{A}^{T} \widetilde{\mathbf{u}}-\left(\mathbf{A}^{T} \mathbf{A}\right)^{-1} \mathbf{H}^{T} \mathbf{C}
\end{gathered}
$$

where $\mathbf{C}=\left\{\left(\mathbf{H}\left(\mathbf{A}^{T} \mathbf{A}\right)^{-1} \mathbf{H}^{T}\right)^{-1} \mathbf{H}\left(\mathbf{A}^{T} \mathbf{A}\right)^{-1} \mathbf{A}^{T} \widetilde{\mathbf{u}}-\mathbf{H}^{T}\left(\mathbf{H}\left(\mathbf{A}^{T} \mathbf{A}\right)^{-1} \mathbf{H}^{T}\right)^{-1} \mathbf{f}\right\}$ and in the matrix form

$$
\left[\begin{array}{cc}
\mathbf{A}^{T} \mathbf{A} & \mathbf{H}^{T} \\
\mathbf{H} & \mathbf{0}
\end{array}\right] \times\left[\begin{array}{l}
\mathbf{u} \\
\boldsymbol{\lambda}
\end{array}\right]=\left[\begin{array}{c}
\mathbf{A} \tilde{\mathbf{u}} \\
\mathbf{f}
\end{array}\right]
$$

Solution of the set of equations (8) gives the vector $\mathbf{u}$ for which the error function reaches the minimum value and the constraint equations (1) are exactly satisfied. Procedure described above was implemented to verify and correct data determined while calculating stress distribution in 2D disc loaded by the concentrated force.

\section{Numerical implementation}

In order to demonstrate the functionality of the proposed verification procedure, a number of trial calculations have been made. With reference to works $[5,6]$ the examples of verification of the plane stress state in a half-plane, loaded by a concentrated force $\mathbf{P}$ at the boundary is presented. Based on [8] theoretical stress distribution $\left(\sigma_{x x}\right.$ and $\left.\sigma_{y y}\right)$ inside the half-plane loaded along the edge can be presented in the form 


$$
\sigma_{x x}=\frac{2 P}{\pi} \frac{x^{3}}{\left(x^{2}+y^{2}\right)^{2}} ; \quad \sigma_{y y}=\frac{2 P}{\pi} \frac{x y^{2}}{\left(x^{2}+y^{2}\right)\left(x^{2}+y^{2}\right)} ;
$$

Force $\mathbf{P}$ was assumed to be equal to $5[\mathrm{kN} / \mathrm{mb}]$. The size of the domain (fig.1) was depicted in $[\mathrm{m}]$ and the stress distribution in $[\mathrm{kPa}]$. Calculations were performed on irregular grid of nodes with equilibrium and continuity equations in the forms:

$$
\frac{\partial^{2} \sigma_{x x}}{\partial x^{2}}-\frac{\partial^{2} \sigma_{y y}}{\partial y^{2}}=0 ; \Delta\left(\sigma_{x x}+\sigma_{y y}\right)=0
$$

The relative error implemented in the tests has taken the form:

$$
\varepsilon_{R}=\left|\frac{\sigma_{\text {exact }}-\sigma}{\sigma_{\text {exact }}}\right| \cdot 100 \%
$$

Irregular distribution of nodes was presented on fig.2. Figure 3 shows the exactly calculated (9) $\sigma_{\mathrm{xx}}$ stress component.

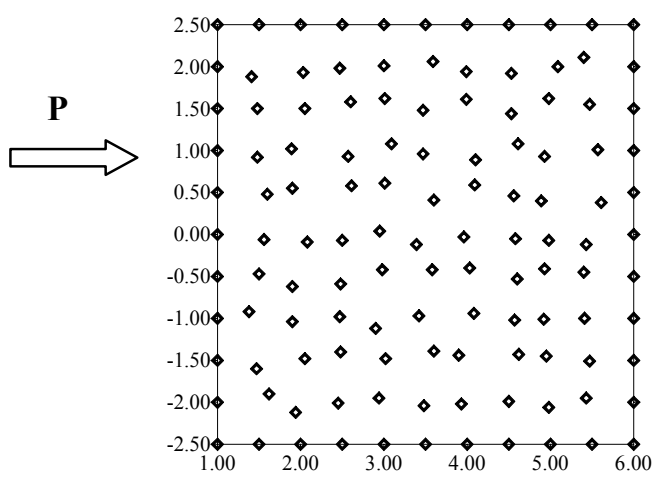

Figure 2: Discretization of the domain.
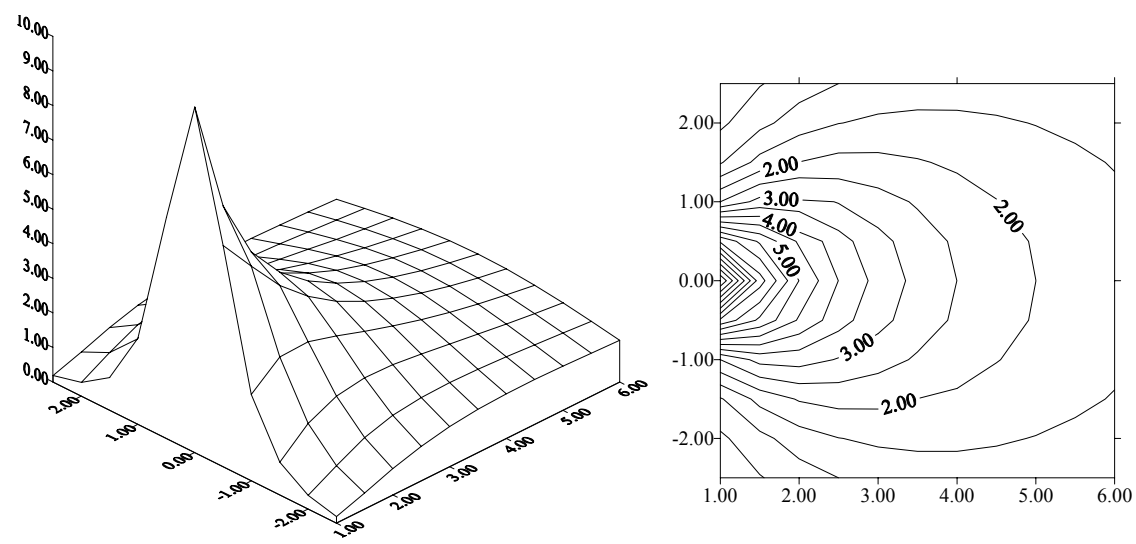

Figure 3: $\quad \sigma_{\mathrm{xx}}$ stress distribution. Input data - exact values. 
To find out the effectiveness of the proposed filtering procedure the above exact solution was artificially disturbed by the local errors reaching up to $50 \%$ of initial values of stresses. Stresses $\sigma_{\text {yy }}$ remained unchanged. Figure 4 shows a view of disturbed stress state and fig. 5 depicts the measure of relative error of corrupted data.

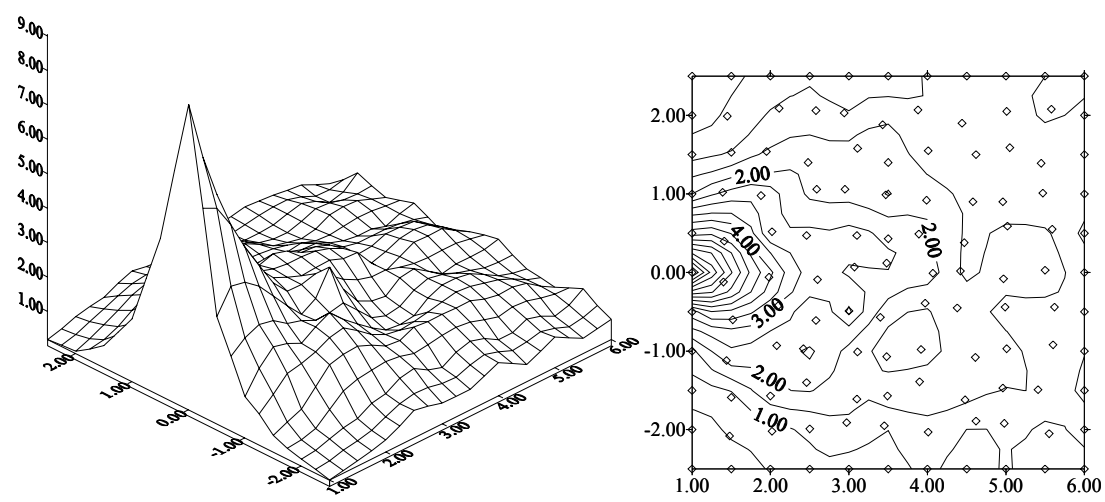

Figure 4: $\quad \sigma_{\mathrm{xx}}$ stress distribution with random errors - corrupted data.

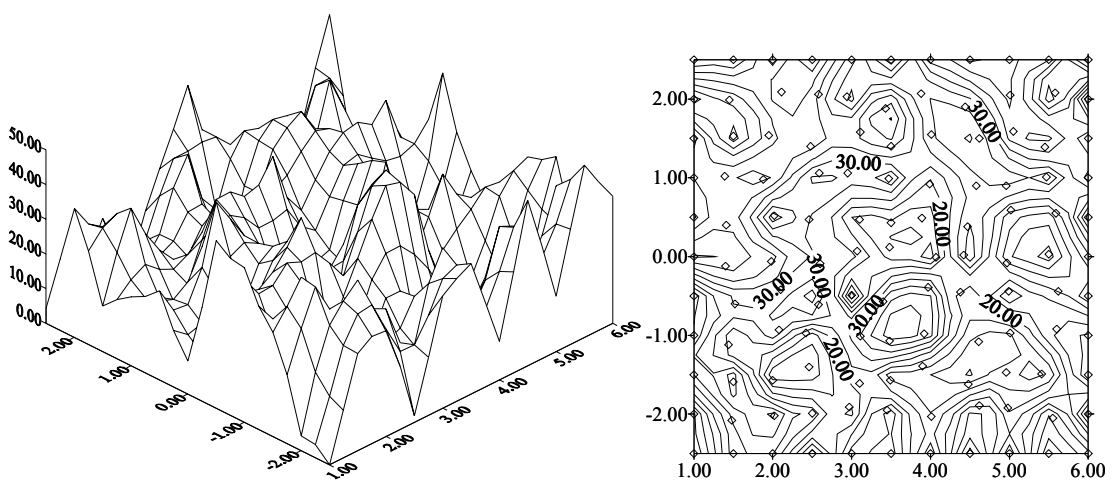

Figure 5: $\quad$ Relative error of $\sigma_{\mathrm{xx}}-$ corrupted data.

Prepared corrupted data were subjected to filtering process with two types of constrained equations applied:

a) constrained equations (10) were imposed only on the internal nodes of the domain;

b) in addition to the previous case the boundary conditions of I order (assumed values of function on the edge of the domain) were introduced.

The results of filtration in first case were presented in fig. 6 and 7 below. One can observe high efficiency of the procedure for the internal nodes and low for the boundary ones (fig.7). This is the effect of FDM approach of constrained equations only on internal nodes of the domain. 


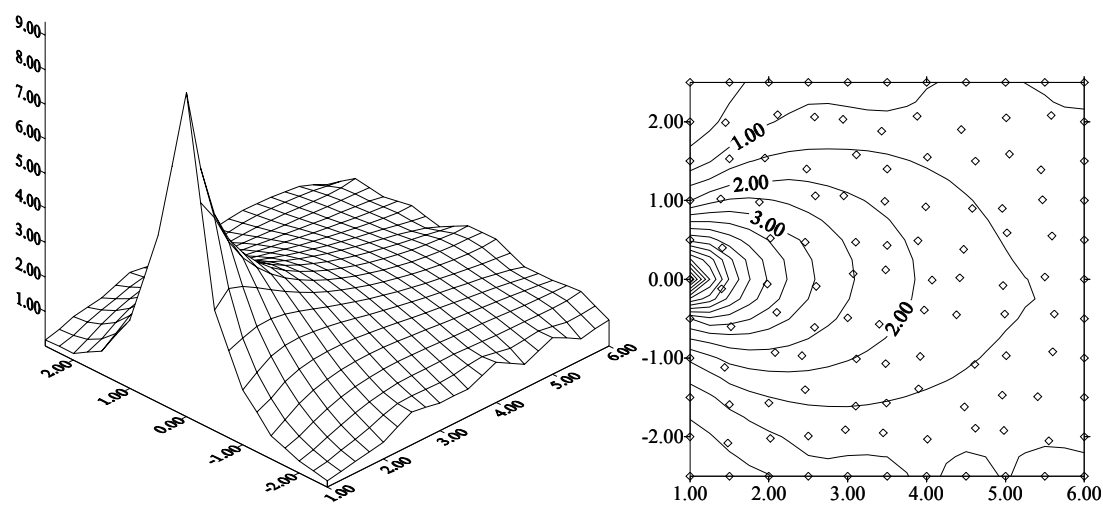

Figure 6: $\quad$ Stress $\sigma_{\mathrm{xx}}$ distribution after filtering. Constrains (10) imposed only on internal nodes of the domain.

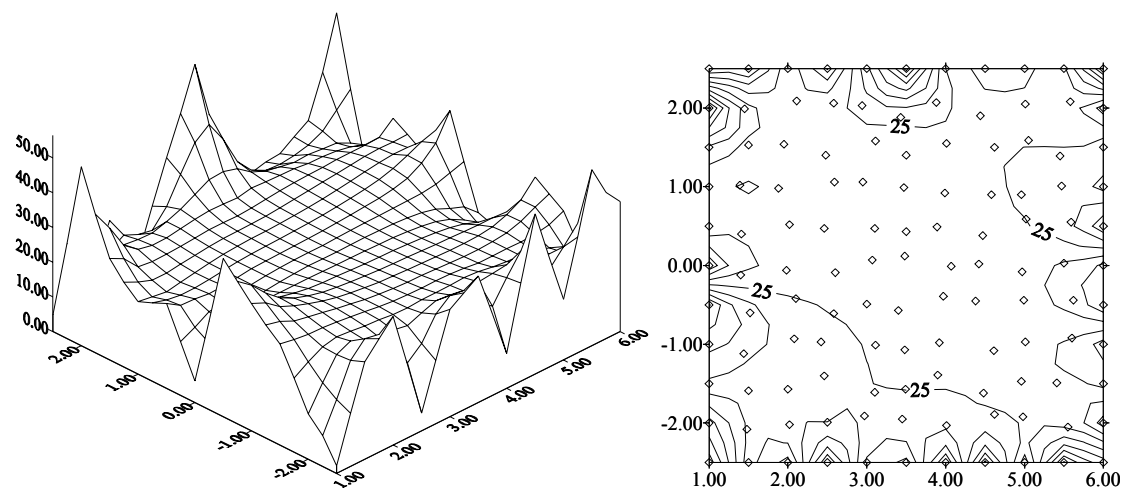

Figure 7: Relative (11) errors of $\sigma_{\mathrm{xx}}$ after filtering of corrupted data. Constrains (10) imposed only on internal nodes of the domain.

Very often certain boundary conditions in the analysed domains are explicitly known and should be imposed within the solution obtained from the filtering process. The result of such a procedure is presented on fig. 8, where theoretically calculated values of the approximated function were applied at the boundary points of analysed domain. It is worthy to point out that the maximum local relative error of $\sigma_{x x}$ was reduced from $50 \%$ to less than $8 \%$ (fig.9).

To estimate the quality of the numerical solution one can calculate the distribution of so called local approach error defined by the equation:

$$
\varepsilon_{\mathrm{L}}=\left|\frac{\sigma^{\text {approx }}-\sigma}{\sigma^{\text {approx }}}\right|
$$

The distribution of such an error was presented in fig. 10 and 11. It is easy to notice, that estimation of the required density of node distribution based on such an approach is destined to fail. 


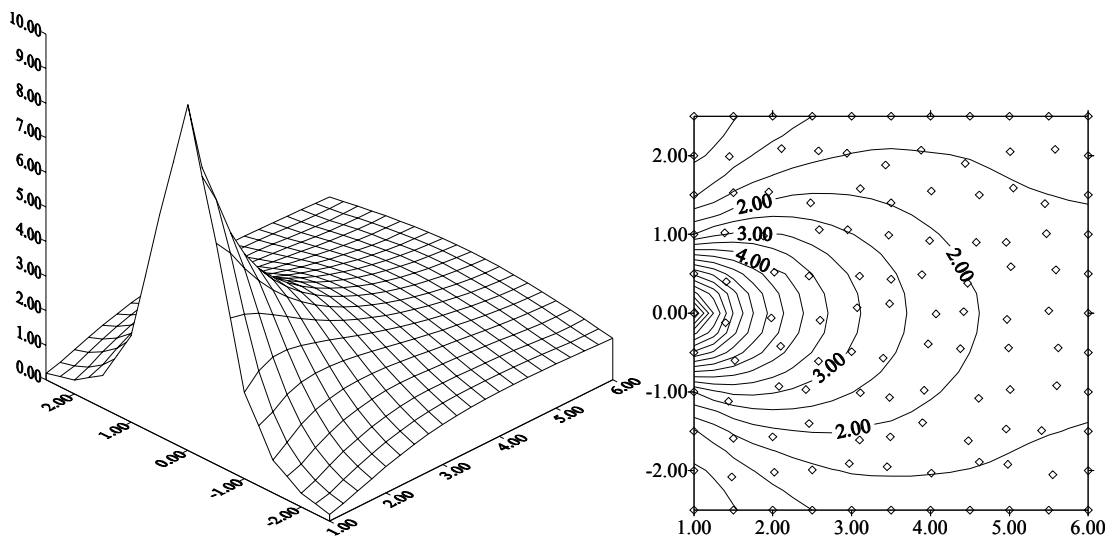

Figure 8: $\quad$ Stress distribution $\sigma_{\mathrm{xx}}$ after filtering of corrupted data. Constrains (10) with boundary condition imposed.

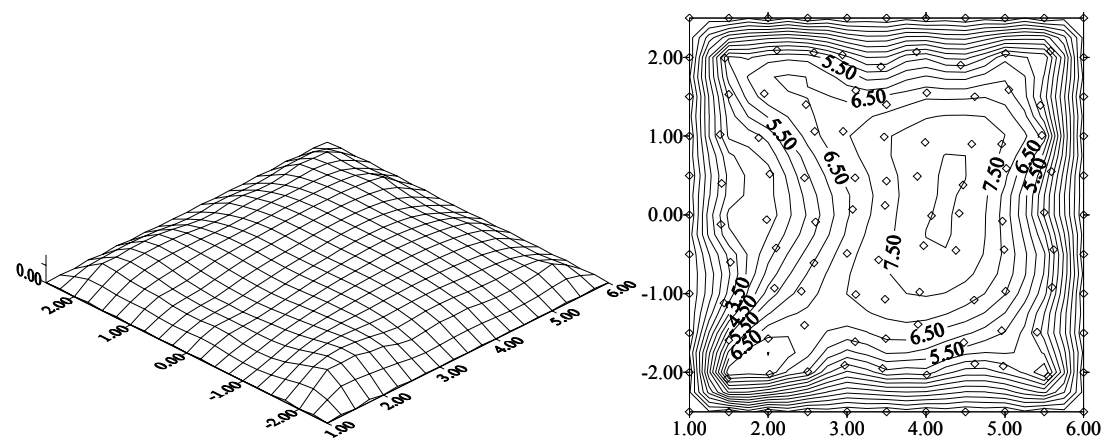

Figure 9: Relative (11) errors of $\sigma_{\mathrm{xx}}$. after filtering of corrupted data. Constrains (10) with boundary condition imposed.

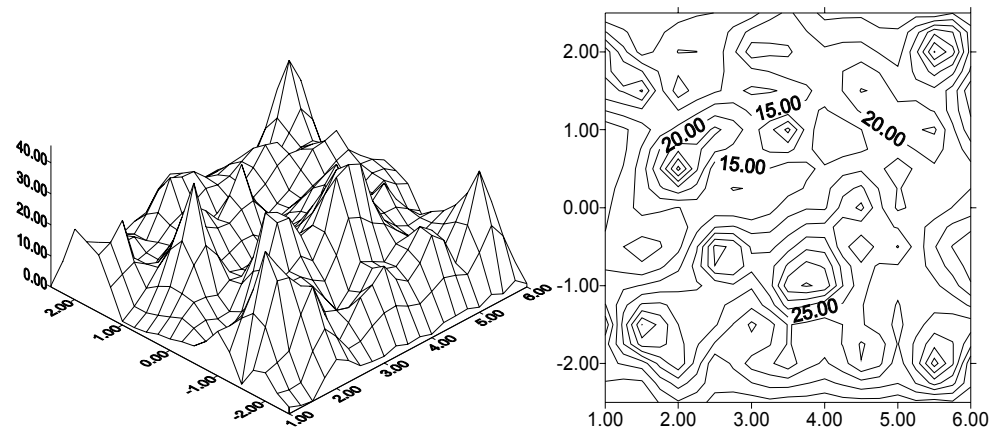

Figure 10: Relative (12) errors of $\sigma_{\mathrm{xx}}$. Constrains (10) imposed on internal nodes of the domain. 


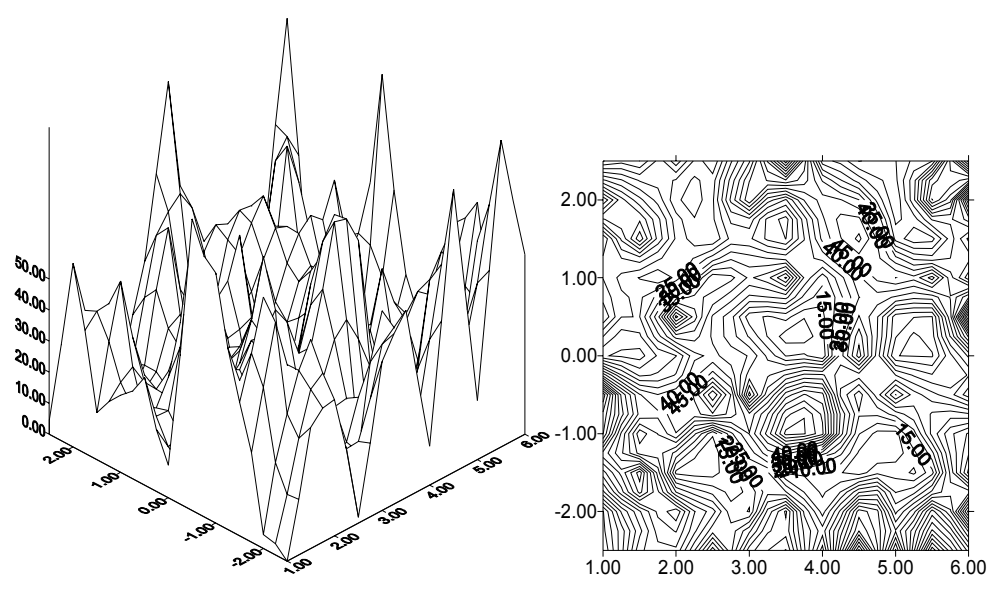

Figure 11: Relative (12) errors of $\sigma_{\mathrm{xx}}$. Constrains (10) with boundary condition imposed.

For further consideration the parameter called "efficiency index" will be introduced and defined by the equation:

$$
\psi=\frac{\|e\|_{\text {approx }}}{\|e\|_{\text {exact }_{i}}}
$$

This parameter equals $\Psi=0.41$ when only constraints (10) are imposed, while when boundary conditions are satisfied $\Psi=0.84$. Efficiency index takes here a role of a measure of approach quality. The perfect situation occurs when the estimated and exact solutions are identical e.g. $\Psi=1.00$. High value of the efficiency index in second case is explained by the fig. 12 where one can note a similar distribution of absolute errors of corrupted and estimated $\sigma_{\mathrm{xx}}$.

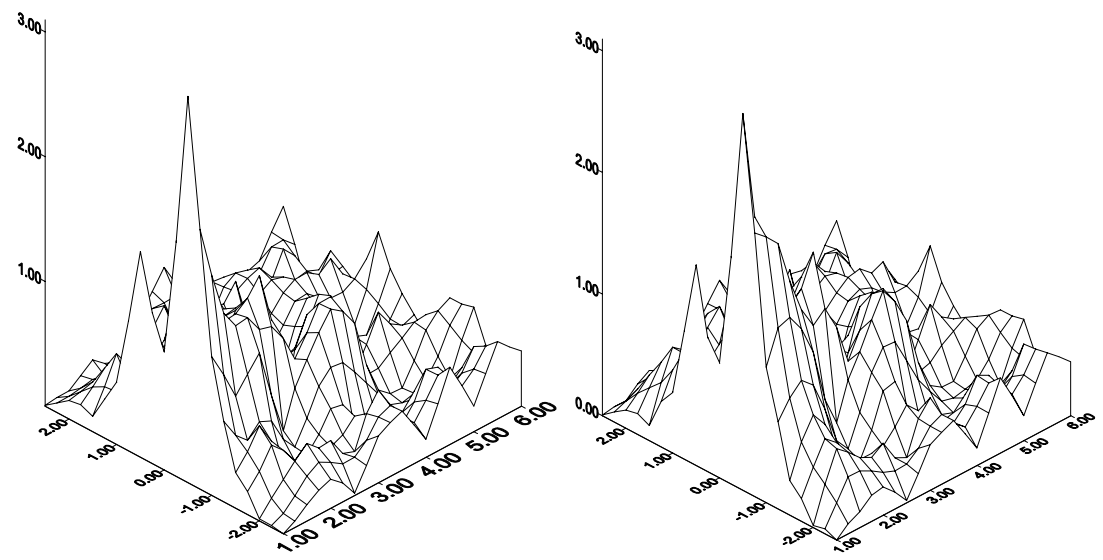

Figure 12: $\quad$ Errors $\left|\sigma^{\text {approx }}-\sigma\right|$ and $\left|\sigma^{\text {exact }}-\sigma\right|$ of $\sigma_{\mathrm{xx}}$. Second case. 
Now we are going to provide a way for expressing the local error in terms of the nodal densities for which one can obtain even error distribution. This is the essential task underlying adaptive technologies. Based on our considerations it turns out that to define such a nodal distribution it is required to apply local as well as global concentration index. Lets introduce:

Local index of nodal density correction defined as:

$$
\bar{\xi}_{\text {oLi }}=\frac{\left|\sigma_{i}^{\text {exact }}-\sigma_{i}^{\text {approx }}\right|}{\|e\|}
$$

Average error in the analysed domain may be calculated as (n number of nodes):

$$
\|e\|_{\sigma}=\frac{1}{\sqrt{n}} \sqrt{\sum_{i=1}^{n}\left(\sigma_{i}^{\text {exact }}-\sigma_{i}^{\text {approx }}\right)^{2}}
$$

Total weighted norm of stresses is depicted by:

$$
\|U\|_{\sigma}=\frac{1}{\sqrt{n}} \sqrt{\left(\sum_{i=1}^{n} \sigma_{i}^{\text {exact }}\right)^{2}}
$$

Finally, global index of nodal density correction, grid refinement, defined as:

$$
\xi_{\sigma}=\frac{\|e\|_{\sigma}}{\eta \cdot\|U\|_{\sigma}}
$$

where $\eta$ corresponds to the level of imposed error distribution.

Based on the norms listed above, global - local index of increasing grid density (I type) may be defined using square of the global index as:

$$
\xi_{\sigma L i}=\xi_{\sigma}{ }^{2} \cdot \bar{\xi}_{\sigma L i}=\left(\frac{\|e\|_{\sigma}}{\eta \cdot\|U\|_{\sigma}}\right)^{2} \cdot \frac{\left|\sigma_{i}^{\text {exact }}-\sigma_{i}^{\text {approx }}\right|}{\|e\|_{\sigma}}=\frac{\|e\|_{\sigma}}{\eta^{2} \cdot\|U\|_{\sigma}^{2}}\left|\sigma_{i}^{\text {exact }}-\sigma_{i}^{\text {approx }}\right|
$$

Other possible global - local index of increasing grid density (II type) may be defined using linear form of global refinement index as

$$
\xi_{\sigma L i}=\xi_{\sigma} \cdot \bar{\xi}_{\sigma L i}=\left(\frac{\|e\|_{\sigma}}{\eta \cdot\|U\|_{\sigma}}\right) \cdot \frac{\left|\sigma_{i}^{\text {exact }}-\sigma_{i}^{\text {approx }}\right|}{\|e\|_{\sigma}}=\frac{1}{\eta \cdot\|U\|_{\sigma}}\left|\sigma_{i}^{\text {exact }}-\sigma_{i}^{\text {approx }}\right|
$$

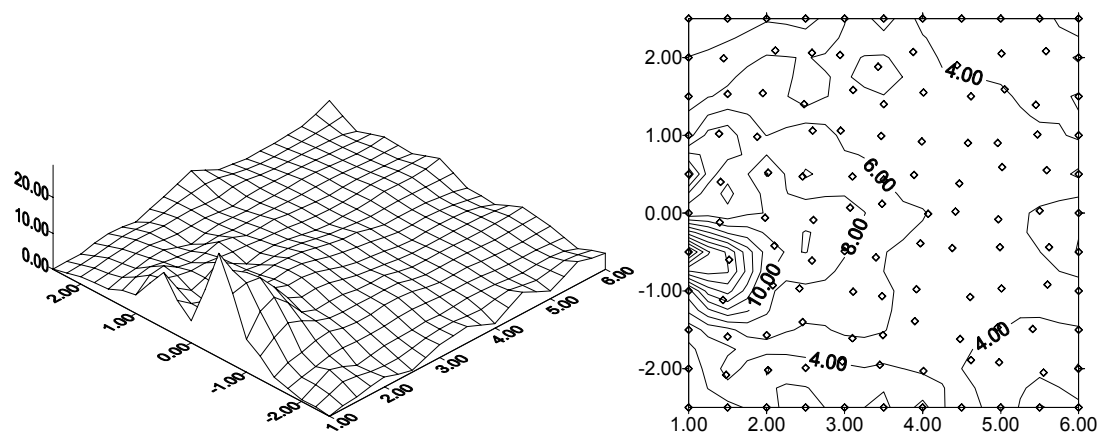

Figure 13: Local-global mesh refinement index (18). Permissible error $\eta=0.10$. 
The distribution of the global - local refinement index (19) is depicted in fig. 14 .

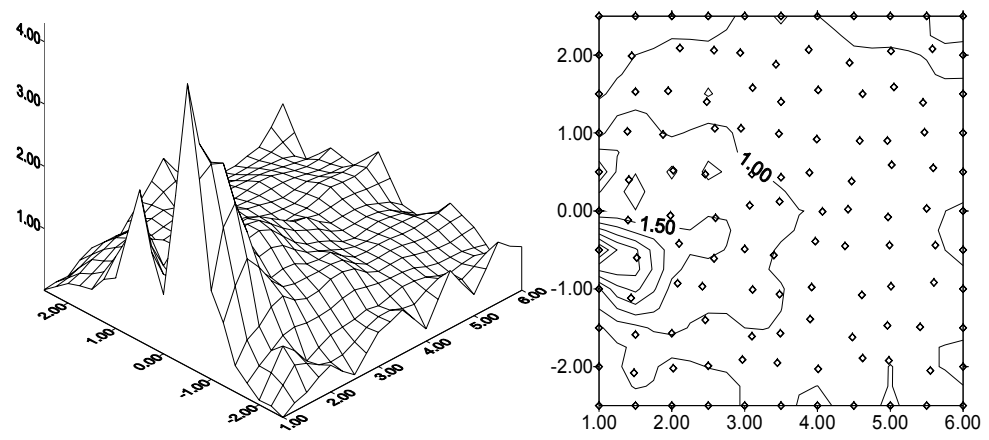

Figure 14: Local-global mesh refinement index (19). Permissible error $\eta=0.10$.

It is easy to observe on the results presented above that the refinement of the grid was necessary within the areas exhibiting high gradient of the sought function. Moreover it is worthy to note that in I-st case, the required grid density was overestimated (20) while in II-nd attempt the refinement index takes realistic value of 4 (verified during analysis).

\section{Conclusions}

In this work the development of an adaptive approach for the experimental as well as numerical data collections was presented. An adaptive procedure of experimental and numerical data collections based on a'posteriori error analysis of data was proposed. It includes estimation of the new grid density taking into account equal distribution of an error (using different error norms). Thus adaptive procedure of experiment or numerical discretization planning is possible.

Numerical approach was executed using Adaptive Meshless Finite Difference Method with certain additional constrains taken into consideration.

The paper also presents a new concept of error estimation with the use of so called global - local estimator. The implementation of such estimators lets to refine the grid of numerical or experimental nodes. Obtained results show a good efficiency of proposed adaptive procedure.

\section{References}

[1] W. Karmowski, J. Orkisz, Physically Based Method of Enhancement of Experimental Data - Concept, Formulation, and Application to Identification of Residual Stresses, Proc. of the IUTAM Symp. on Inverse Problems in Engng Mech., Tokyo, Japan, Springer-Verlag, 1993, 61-70. 
[2] J. Krok, An Extended Approach to Error Control in Experimental and Numerical Data Smoothing and Evaluation Using the Meshless FDM, Revue Europenne des elements finis, no 7-8/2002, pages 913-935.

[3] J. Krok, Meshless FDM based Approach to Error Control and Evaluation of Experimental or Numerical Data. II MIT Conf. on Comp. Fluid and Solid Mechanics, 2003, Cambridge, MA, USA.

[4] J. Krok, J. Wojtas, An Adaptive Approach to Experimental Data Collection Based on A Posteriori Error Estimation of Data, Comp. Meth. in Mechanics - CMM-2007, June 2007, Spała-Łódź, pp.1-13.

[5] S. A. Łukasiewicz, M. Stanuszek, J.A. Czyż, Filtering of the Experimental or FEM Data in Plane Stress and Strain Fields, Experimental Mechanics, 1993, June, pp. 139-147.

[6] S. A. Łukaszewicz, M. Stanuszek, Constrained, weighted, least square technique for correcting experimental data, 6th Int. Conf. on Comp. Methods and Experimental Measurements 93, Vol 2: Stress analysis, pp. 467-480, Elsevier Applied science, London New York, 1993.

[7] J. Magiera, Non-statistical physically reasonable technique for a posteriori estimation of experimental data error, Computer Assisted Mechanics and Engineering Sciences, 13, 593-611, 2006

[8] S. Timoshenko, J. N. Goodier Theory of elasticity, New York Toronto London, 1951.

[9] O.C. Zienkiewicz, R.L. Taylor, The Finite The Finite Element Method, Vols. I-III, Sixth ed. Butterworth-Heinemann, Oxford, 2005. 